Tropical Journal of Pharmaceutical Research November 2021; 20 (11): 2311-2316

ISSN: $1596-5996$ (print); 1596-9827 (electronic)

(C) Pharmacotherapy Group, Faculty of Pharmacy, University of Benin, Benin City, 300001 Nigeria

Available online at http://www.tjpr.org

Original Research Article

http://dx.doi.org/10.4314/tjpr.v20i11.12

\title{
Clinical effect of astragaloside IV on breast carcinoma cells based on MDR1: A randomised trial
}

\author{
Liangdong Chen ${ }^{1 *}$, Deqiang Zhuo ${ }^{2}$, Hongyin Yuan ${ }^{1}$ \\ ${ }^{1}$ Department of Thyroid and Breast Surgery, ${ }^{2}$ Department of Radiology, Zhongnan Hospital of Wuhan University, \\ Wuhan430071, Hubei Province, China \\ *For correspondence: Email: cp8ly4@163.com; Tel: +86-13871419532 \\ ${ }^{7}$ Chen $L$ and Zhuo $D$ are co-first authors
}

Sent for review: 31 May 2021

Revised accepted: 27 October 2021

\begin{abstract}
Purpose: To study the clinical effect of astragaloside IV on breast carcinoma cells (BCCs), and its potential mechanisms with respect to multiple drug resistance-1 (MDR1)

Methods: The cytotoxicity of astragaloside IV to BCCs was determined using CCK-8 test, and values of its half inhibitory concentration $\left(I_{50}\right)$ were determined. Transwell assay and flow cytometry were performed to determine the effect of astragaloside $(13 \mu \mathrm{g} / \mathrm{mL})$ on cell invasion and apoptosis. The contents of MDR1 mRNA in BC tissues and cells were determined using real-time quantitative polymerase chain reaction (qRT-PCR), while the protein expression levels of MDR1 in BC cells were determined using western blot assay.

Results: The IC 50 of astragaloside IV for MCF-7 and MDA-MB-231 BCCs were $12.57 \mu \mathrm{g} / \mathrm{mL}$ and 13.91 $\mu \mathrm{g} / \mathrm{mL}$, respectively. Transwell experiment showed significantly inhibited invasive capacity and enhanced apoptotic potential of the BCCs after astragaloside IV intervention. However, invasive capacities of the BCCs were markedly enhanced, while their apoptotic capacities were inhibited after transfection with si-MDR1, when compared with controls $(p<0.05)$. Results of $q R T-P C R$ revealed that the mRNA content of MDR1 in BC tissues and cells $(0.42 \pm 0.11)$ was significantly lower than that in normal tissues (0.95 $\pm 0.18 ; p<0.05)$. Results from western blot assay revealed that the relative expression levels of MDR1 protein were decreased, with values of $0.21 \pm 0.05,0.32 \pm 0.07$ and $0.74 \pm 0.15$ for MCF-10A, MCF-7, MAD-MB-231 and MCF-10A, respectively $(p<0.05)$.

Conclusion: Astragaloside IV regulates the metastasis and apoptosis of BCCs through regulation of MDR1. It also inhibits cell invasion but enhances the apoptosis of BC cells transfected with si-MDR1. These results highlight the prospects of the compound for the treatment of $B C$.
\end{abstract}

Keywords: Astragaloside IV, Multi-drug resistance gene, Breast carcinoma, Metastasis, Apoptosis

This is an Open Access article that uses a funding model which does not charge readers or their institutions for access and distributed under the terms of the Creative Commons Attribution License (http://creativecommons.org/licenses/by/4.0) and the Budapest Open Access Initiative (http://www.budapestopenaccessinitiative.org/read), which permit unrestricted use, distribution, and reproduction in any medium, provided the original work is properly credited.

Tropical Journal of Pharmaceutical Research is indexed by Science Citation Index (SciSearch), Scopus, International Pharmaceutical Abstract, Chemical Abstracts, Embase, Index Copernicus, EBSCO, African Index Medicus, JournalSeek, Journal Citation Reports/Science Edition, Directory of Open Access Journals (DOAJ), African Journal Online, Bioline International, Open-J-Gate and Pharmacy Abstracts

\section{INTRODUCTION}

Breast cancer $(B C)$ is among the most predominant malignancies, with excessively high morbidity among females, when compared to males [1]. Indeed, BC is considered an essential public health issue. Data from a recent survey have confirmed curability in $80 \%$ of early nonmetastatic BC cases [2]. Currently, the combination of surgery, radiotherapy and chemotherapy is the gold standard for the treatment of $\mathrm{BC}$ [3]. However, this treatment 
strategy does not result in satisfactory efficacy, and it does not ameliorate the poor prognosis of patients after metastasis. Therefore, there is need for investigations on the potential mechanism involved in the pathogenesis of $\mathrm{BC}$, with a view to developing better treatment methods for the disease.

Astragalus, a traditional Chinese medicinal herb, is the dried root of Bunde or Fabaceae. Astragaloside IV, the main bioactive component of astragalus extract, has been widely used to regulate the immune system and provide neuroprotection. A recent research has also revealed its inhibitory effect on tumor growth and metastasis [4]. For example, astragaloside IV suppressed the aggravation and metastasis of lung carcinoma by regulating macrophage polarization via AMPK signaling, and it inhibited the invasion and metastasis of SiHA cervical carcinoma cells through PI3K and MAPK pathways mediated by TGF- $\beta 1$ [5].

It has been reported that astragaloside IV regulated the transcription of multidrug resistance (MDR) gene. The MDR-1 gene encodes P-glycoprotein, an ATP-binding cassette $(A B C)$ transporter which plays the role of an energy-dependent efflux pump on the plasma membrane [6]. Wang et al [7] found that astragaloside IV is involved in the occurrence of hepatocellular carcinoma through regulation of MDR1. However, the role of astragaloside IV in the occurrence $B C$ is still not elucidated. The role of astragaloside IV in $\mathrm{BC}$, and the associated mechanism, were investigated in this study, with the aim of providing clinical evidence necessary for development of novel treatment methods for BC.

\section{METHODS}

\section{GEPIA2 database analysis}

The expression of MDR1 in BC was retrieved from the GEPIA2 database analysis. The following steps were taken on the website of GEPIA2 (http://gepia2.carcinomapku.cn/\#analysis) [8]: After logging in, the following items on the website were selected and clicked in sequence, namely, Expression Analysis, Expression DIY, Box Plot, ABCB1, |Log2FC| Cutoff:1, p-value Cutoff:0.01, BRCA, Match TCGA normal and GTEx data, and Plot.

\section{Clinical profiles of patients}

A total of $30 \mathrm{BC}$ cases treated in Zhongnan Hospital of Wuhan University were identified. During surgery, samples were collected from malignant tissues and nearby tissues and kept in liquid nitrogen prior to examination. The TNM stages of the patients were I-III, comprising 15 cases in stage I, 9 in stage II, and 6 in stage III. The patients had no other tumors. Approval for this investigation was received from the medical ethical committee of our institution. The research was done in line with Helsinki Declaration [9].

\section{Cell cultivation}

The BCCs i.e., MCF-7 and MDA-MB-231, and MCF-10A human normal breast epithelial cells (ATCC, Manassas, VA) were cultured in RPMI1640 medium containing $10 \%$ FBS (Invitrogen), penicillin $(100 \mathrm{U} / \mathrm{ml})$ and streptomycin (100 $\mu \mathrm{g} / \mathrm{ml}$ ) (Sigma-Aldrich) at $37{ }^{\circ} \mathrm{C}$ in a $5 \% \mathrm{CO}_{2}$ atmosphere. In order to determine the toxicity of astragaloside IV, BCCs were cultured with varying doses of the drug $(5,10,25$, and 50 $\mu \mathrm{g} / \mathrm{mL})$. Then, its effect on the growth ability of cells was determined by incubating the cells with the drug at its $\mathrm{IC}_{50}$.

\section{Cell transfection}

A member of the sirtuin family of small interfering RNA (siRNA), MDR1 was purchased from Invitrogen (Carlsbad, California, USA). The cells were treated with Lipofectamine 2000 (Invitrogen). When the cell density reached $60-$ $70 \%$, siRNA (50 nmol/L) was transfected with liposome.

\section{Cell proliferation}

Cell proliferation was evaluated with CCK-8. The BCCs were inoculated in a 96-well plate $\left(1 \times 10^{4}\right.$ cells/well), and then exposed to varying doses of astragaloside IV for $48 \mathrm{~h}$. Subsequently, CCK-8 reagent was added to each well and incubated for $30 \mathrm{~min}$, followed by oscillation on an oscillator for $30 \mathrm{sec}$. Optical density was read in a Bio-Rad 680 spectrophotometer.

\section{qRT-PCR}

Total RNA was extracted with TRIzol reagent purchased from Shanghai Beyotime Biotechnology Co. Ltd. Complementary DNA (cDNA) was synthesized using TaqMan reverse transcription reagent (Life Technologies). The fluorescence quantification was conducted using SYBR Green Premix Ex Taq II for amplification. The expression level of MDR1 was determined quantitatively, with GAPDH as housekeeping gene. The primers used were purchased from TaKaPa (Japan). The relative expressions of the genes were calculated using $2^{-\Delta \Delta C T}$. The primer 
sequences of MDR1 and GAPDH are shown in Table 1.

Table 1: Primer sequences of MDR1 and GAPDH

\begin{tabular}{ll}
\hline & MDR1 \\
\hline Upstream & 5'-CCCATCATTGCAATAGCAGG-3' \\
Downstream & 5'-GTTCAAACTTCTGCTCCTGA-3' \\
\hline
\end{tabular}

\section{Western blot assay}

The cells were subjected to low-speed centrifugation, and the cell mass was taken up in ice-cold PBS, and subjected to low-speed centrifugation on a vortex mixer at $3000 \mathrm{rpm}$ for 2 - 3 min. Then, an equivalent volume of PBS was added to the cells after removal of the supernatant, prior to vortex oscillation of the cell suspension. The cells were then lysed by mixing with equivalent volume of cell lysis buffer and quickly transferred to an ice-cold collection tube, followed by centrifugation at 14000-16000 rpm for $30 \mathrm{sec}$. Subsequently, the spin column was discarded and the collection tube was placed on ice to obtain the extracted protein which was then used for the downstream experiment. The protein was quantified and resolved via SDSPAGE and electro-transferred to PVDF membrane. The membrane was blocked by incubation with $5 \%$ BSA, followed by incubation at $4{ }^{\circ} \mathrm{C}$ overnight with primary antibodies i.e., anti- $\beta$-actin and anti-MDR1 (which had been diluted beforehand with TBST). On the next day, the membrane was rinsed, prior to incubation for $1 \mathrm{~h}$ at room temperature with secondary antibodies labeled with horseradish peroxidase (HRP). The intensity of each protein band was determined using the optical density method, while analysis was done using Image $\mathrm{J}$ software (NIH).

\section{Determination of cell invasion}

Invasion assay was conducted in Transwell chambers coated with Matrigel membrane. A $100-\mu \mathrm{L}$ cell suspension $\left(5 \times 10^{4}\right.$ cells) prepared in serum-free medium was put in the upper chamber, while the medium including $10 \%$ FBS was placed in the lower chamber as a chemical attractant. The cells were cultivated for $48 \mathrm{~h}$, and the non-invasive cells above the filter were discarded. Cells that invaded the bottom surface were fixed with $4 \%$ paraformaldehyde and stained with hematoxylin. Four random fields were selected for observation of the cells under a microscope $(\times 100)$.

\section{Determination of apoptosis}

Digested cells were rinsed with PBS, centrifuged at $2000 \mathrm{rpm}$ for $5 \mathrm{~min}$, and $5 \times 10^{5}$ cells were collected. Cell suspension $(500 \mu \mathrm{L})$ was mixed with Annexin V-FITC $(5 \mu \mathrm{L})$ and PI $(5 \mu \mathrm{L})$, and mixed well. The cells were cultivated at $20-25^{\circ} \mathrm{C}$ for 10 min in the dark. Finally, FACSCanto II was used for flow cytometry, and the data were anfAREs with Flow lo 94 software

5'-GTGAAGGTCGGTGTCAACGGATTT-3'

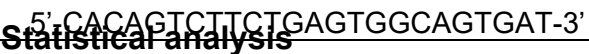

Data analysis was done with SPSS 19.0. GraphPad Prism was applied to visualize data pictures. Counting data are presented as numbers and percentages [n (\%)] and analyzed using $\mathrm{X} 2$. Measurement data are presented as mean $\pm S D$, and were compared with $t$-test. Multi-group comparison was done with one-way ANOVA. Values of $p<0.05$ are indicative of significant differences.

\section{RESULTS}

\section{Toxicity of astragaloside IV in BCCs}

In this study, the evaluation of the toxicity of astragaloside IV to BC cells was conducted via treatment of $\mathrm{BC}$ cells with astragaloside IV at doses of $0.1-50 \mu \mathrm{g} / \mathrm{mL}$. After $48 \mathrm{~h}$, the viability of BC cells was effectively inhibited in a concentration-dependent manner (Figure $1 \mathrm{~A}$ ). Further analysis showed that $\mathrm{IC}_{50}$ values of astragaloside IV in BCCs were $12.57 \mu \mathrm{g} / \mathrm{mL}$ and $13.91 \mu \mathrm{g} / \mathrm{ml}$ (Figure $1 \mathrm{~B}$ ). Thus, astragaloside IV at the concentration of $13 \mu \mathrm{g} / \mathrm{mL}$ was used for the treatment of the cells in the measurement of changes in cell viability.
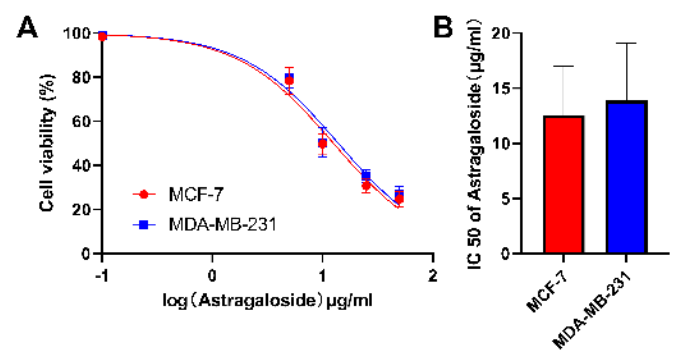

Figure 1: Toxicity of astragaloside IV in BC cells. A: CCK-8 test was applied to examine the toxicity of astragaloside IV to the $B C$ cells. $B$ : $I_{50}$ values of astragaloside IV in BC cells

\section{Astragaloside IV inhibited the apoptosis induced by the invasion of $B C$ cells}

Transwell experiment revealed marked inhibition of the invasion of $\mathrm{BC}$ cells, relative to the controls (Figure $2 \mathrm{~A}$ ), while flow cytometry demonstrated that apoptosis of $\mathrm{BC}$ cells was markedly enhanced, relative to the control (Figure $2 \mathrm{~B}$ ). These results indicate that astragaloside

Trop J Pharm Res, November 2021; 20(11): 2313 
inhibited metastasis of BC cells and promoted their apoptosis.

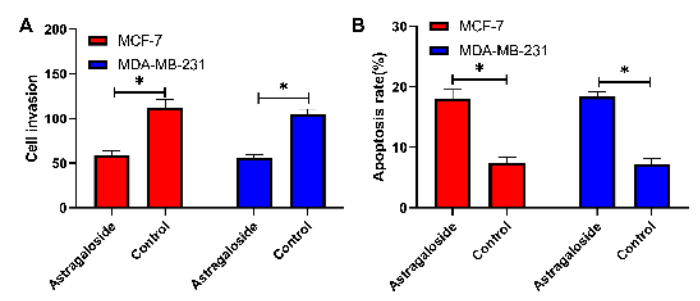

Figure 2: Effect of astragaloside IV on metastasis and apoptosis of BCCs. A: Transwell experiment showed that astragaloside IV effectively inhibited the invasion of BCCs. B: Flow cytometry showed that astragaloside IV induced apoptotic changes in BCCs. ${ }^{*} P<0.05$

\section{MDR1 was highly expressed in BCCs}

The BC expressions of MDR1 in TCGA and GTEx databases were analyzed in the GEPIA2 database. The results of analysis revealed low expression of MDR1 in BCCs (Figure $3 \mathrm{~A}$ ). Moreover, there were drastically reduced $\mathrm{mRNA}$ levels of MDR1 in BCCs and tissues (Figures $3 \mathrm{~B}$ \& $3 \mathrm{C}$ ). Western blot assay also confirmed the decrease in MDR1 expression in BCCs. The above results suggest that MDR1 may be involved in the occurrence and development of BC.
A

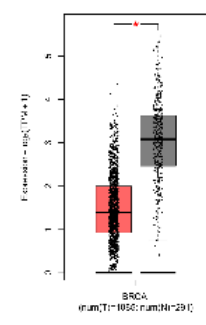

C

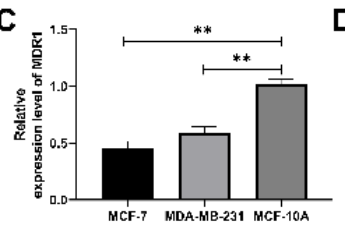

B

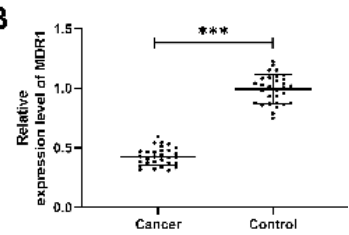

D

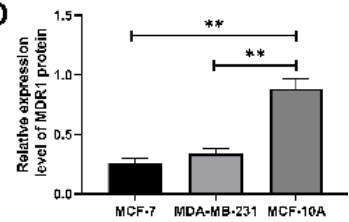

Figure 3: Expression of MDR 1 in BC. A: GEPIA2 online database was applied to predict MDR1 expression in BC. B: Relative MDRI mRNA levels in $B C$ and nearby tissues. C: Relative MDRI mRNA levels in BCCs. D: Relative protein levels of MDR1 in BCCs. ${ }^{* \star} P<0.01,{ }^{* * *} p<0.001$

\section{Knock-down of MDR1 promoted cell invasion and inhibited apoptosis of BC cells}

The content of MDR1 in BC cells was decreased (Figures $4 \mathrm{~A}$ and $4 \mathrm{~B}$ ). Transwell experiment revealed that the invasion of $B C$ cells transfected with si-MDR1 was significantly enhanced, when compared with the control (Figure $4 \mathrm{C}$ ), while apoptosis of BC cells subjected to transfection with si-MDR1 was significantly reduced, when compared with the control (Figure 4 D). Therefore, MDR1 knockout promoted the metastasis of BC cells and inhibited their apoptosis.
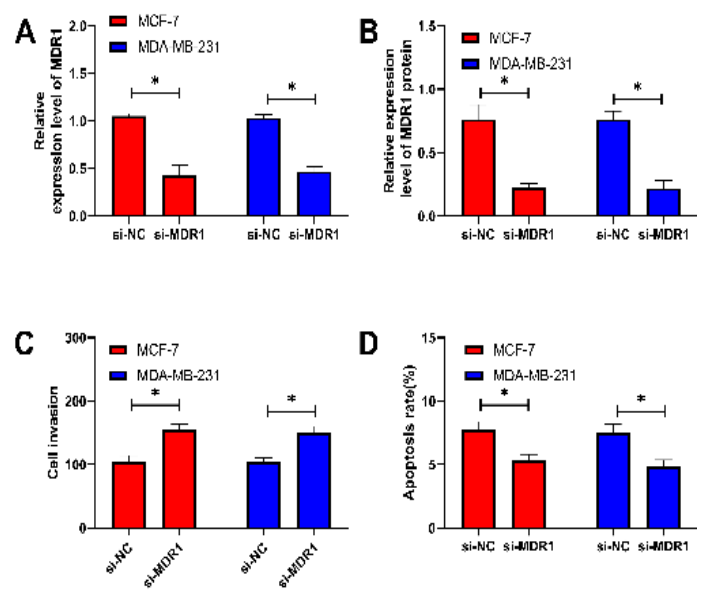

Figure 4: si-MDR1 promoted invasion and inhibited apoptosis of BCCs. A: mRNA level of MDR1 in BCCs after transfection with si-MDR1. B: Results of western blot assay applied to determine protein levels of MDR1 in BCCs after transfection with si-MDR1. C. Results of Transwell experiment applied to determine changes in invasion of BCCs after transfection with si-MDR1. D: Changes in apoptosis of BC transfected with si-MDR1. ${ }^{*} P<0.05$

Astragaloside IV inhibited invasion and enhanced apoptotic changes in cells due to transfection with si-MDR1

Astragaloside IV at a concentration of $13 \mu \mathrm{g} / \mathrm{mL}$ was co-cultured with $\mathrm{BC}$ cells transfected with siMDR1 (Figures $5 \mathrm{~A}$ and $5 \mathrm{~B}$ ). It was found that astragaloside IV significantly inhibited the invasion of BCCs following transfection with siMDR1 (Figure $5 \mathrm{C}$ ). In addition, astragaloside IV significantly increased the apoptosis of the BCCs after the transfection with si-MDR1 (Figure $5 \mathrm{D}$ ). Results in Figure 5 demonstrate that astragaloside IV inhibited the metastasis of BC cells and induced apoptosis by up-regulating MDR1.

\section{DISCUSSION}

Notwithstanding advances in the treatment methods for $\mathrm{BC}$, patients with $\mathrm{BC}$ still experience somber prognosis. Results of this study showed that astragaloside IV inhibited metastasis and promoted apoptosis of $\mathrm{BC}$ cells by regulating MDR1, which demonstrates great potential in the treatment of $\mathrm{BC}$. Astragaloside IV is a bioactive 
A

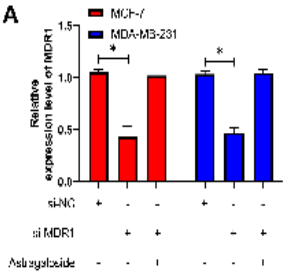

C

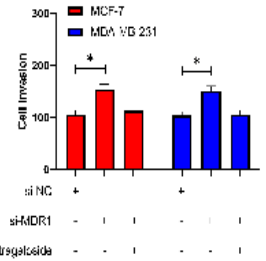

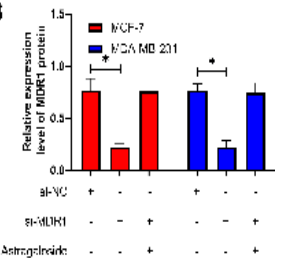

D

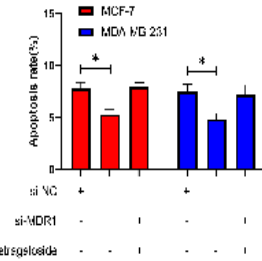

Figure 5: Astragaloside IV regulated MDR1 and reversed metastasis and apoptosis of $B C$ cells. A: Relative mRNA levels of MDR1 after co-culture of astragaloside IV and BC cells transfected with siMDR1. B: Relative protein levels of MDR1 after coculture of astragaloside IV and BCCs following transfection with si-MDR1. C: Changes in cell invasion capacities after co-culture of astragaloside IV and BCCs following transfection with si-MDR1. D: Change in apoptosis after co-culture of astragaloside IV and BC cells transfected with si-MDR1. ${ }^{*} P<0.05$

compound derived from astragalus. It has been confirmed that this bioactive compound produces anti-inflammatory, antioxidant, hypoglycemic and anti-tumor effects [10]. Moreover, it suppressed the proliferative potential of colon carcinoma cells by down-regulating B7-H3, and it inhibited gastric carcinoma cells by blocking the EMT of $\mathrm{PI3K} / \mathrm{AKT} / \mathrm{NF}-\mathrm{KB}$ in gastric cancer [11]. In addition, astragaloside IV plays an important sensitizing role in tumor drug resistance [12]. The results of this study revealed that astragaloside IV inhibited the viability of BCCs in a concentration-dependent fashion. Previous research has also indicated that astragaloside IV suppressed the viabilities of BCCs, which is consistent with the results obtained in this study. Moreover, the $\mathrm{IC}_{50}$ of astragaloside IV in BC was analyzed to further find out the effect of astragaloside IV on metastasis and apoptosis of BCCs. Subsequently, astragaloside IV was used to treat the BCCs at a concentration of $13 \mu \mathrm{g} / \mathrm{mL}$. It was found that astragaloside IV effectively hindered the metastasis of BCCs and induced apoptosis.

Previous research showed the participation of astragaloside IV in tumor growth, metastasis, and drug resistance through regulatory genes. To be specific, astragaloside IV enhanced the chemotherapy sensitivity of colorectal carcinoma by regulating $\mathrm{NOTCH}$, and it inhibited the progression of hepatocellular carcinoma by

regulating the miR-150-5p/ $\beta$-catenin axis [13]. In recent years, MDR1 has become an important gene in tumor research, and it has been reported to inhibit tumor growth [14]. A previous study revealed low expression of MDR1 in BCCs [15]. In this study, the content of MDR1 in BCCs was analyzed through the GEPIA2 database, and it was found that in TCGA and GTEx tumor databases, MDR1 was poorly expressed in BC. Then, results from qRT-PCR showed low expressions of MDR1 in tumor tissues and cells. Results of the western blot experiment also confirmed significantly decreased levels of MDR1 in BCCs, which is consistent with previous results. Furthermore, it was found in this study that knocking down of MDR1 markedly promoted the invasion of BCCs and inhibited apoptosis, which is indicative of the involvement of MDR1 in the metastasis and apoptosis of BCCs. Astragaloside IV was co-cultured with BC cells transfected with si-MDR1, to verify the regulatory effect of astragaloside IV on MDR1 and its role in $\mathrm{BC}$ cell metastasis and apoptosis. The results showed that astragaloside IV suppressed the invasion, and enhanced the apoptosis of $\mathrm{BC}$ cells transfected with si-MDR1, suggesting that astragaloside IV regulated the metastasis and apoptosis of BC cells through MDR1.

However, there are some limitations. Firstly, MDR1 acts in regulating multidrug resistance of BC cells, but this study did not determine whether astragaloside IV could improve drug resistance of $\mathrm{BC}$ cells by regulating MDR1. Secondly, earlier studies have shown that MDR1 was regulated by microRNA and IncRNA, which also underscores the significance of the establishment of a ceRNA network concerning astragaloside IV to monitor its effect on the drug resistance of BCCs [16].

\section{CONCLUSION}

Astragaloside IV regulates the metastasis and apoptosis of BC cells via MDR1. Therefore, it is a potential treatment strategy for $\mathrm{BC}$.

\section{DECLARATIONS}

\section{Conflict of Interest}

No conflict of interest associated with this work.

\section{Contribution of Authors}

We declare that this work was done by the authors named in this article, and all liabilities pertaining to claims relating to the content of this article will be borne by the authors. Liangdong 
Chen and. Deqiang Zhuo conceived and designed the study, collected, analyzed and interpreted the experimental data, and drafted the manuscript; they contributed equally to this work. Hongyin Yuan revised the manuscript for important intellectual content. All authors read and approved the final manuscript.

\section{Open Access}

This is an Open Access article that uses a funding model which does not charge readers or their institutions for access and distributed under the terms of the Creative Commons Attribution License (http://creativecommons.org/licenses/by/ 4.0) and the Budapest Open Access Initiative (http://www.budapestopenaccessinitiative.org/rea d), which permit unrestricted use, distribution, and reproduction in any medium, provided the original work is properly credited.

\section{REFERENCES}

1. Coughlin SS. Epidemiology of Breast Cancer in Women. Adv Exp Med Biol 2019; 1152: 9-29.

2. Harbeck N, Gnant M. Breast cancer. Lancet 2017; 389: 1134-1150.

3. Krug D, Baumann R, Budach W, Dunst J, Feyer $P$, Fietkau R, Haase W, Harms W, Hehr T, Piroth MD, et al. Individualization of post-mastectomy radiotherapy and regional nodal irradiation based on treatment response after neoadjuvant chemotherapy for breast cancer: A systematic review. Strahlenther Onkol 2018; 194: 607618.

4. Li B, Wang F, Liu N, Shen W, Huang T. Astragaloside IV inhibits progression of glioma via blocking MAPKJERK signaling pathway. Biochem Biophys Res Commun 2017; 491: 98-103.

5. Zhang L, Zhou J, Qin X, Huang H, Nie C. Astragaloside $I V$ inhibits the invasion and metastasis of SiHa cervical cancer cells via the TGFbeta1mediated PI3K and MAPK pathways. Oncol Rep 2019; 41: 2975-2986.
6. Bossennec M, Di Roio A, Caux C, Menetrier-Caux C. MDR1 in immunity: friend or foe? Oncoimmunology 2018; 7: e1499388.

7. Wang $P P, X u D J$, Huang $C$, Wang WP, $X U$ WK. Astragaloside reduces the expression level of $P$ glycoprotein in multidrug-resistant human hepatic cancer cell lines. Mol Med Rep 2014; 9: 2131-2137.

8. Tang Z, Kang B, Li C, Chen T, Zhang Z. GEPIA2: an enhanced web server for large-scale expression profiling and interactive analysis. Nucleic Acids Res 2019; 47: W556-W560.

9. Issue Information-Declaration of Helsinki. J Bone Miner Res 2018; 33: BM i-BM ii.

10. Wang $F$, Zhao $Y$, Chen $S$, Chen L, Sun L, Cao M, Li C, Zhou $X$. Astragaloside IV Alleviates Ammonia-Induced Apoptosis and Oxidative Stress in Bovine Mammary Epithelial Cells. Int J Mol Sci 2019; 20.

11. Zhu J, Wen K. Astragaloside IV inhibits TGF-beta1induced epithelial-mesenchymal transition through inhibition of the PI3K/Akt/NF-kappaB pathway in gastric cancer cells. Phytother Res 2018; 32: 1289-1296.

12. Qu X, Gao H, Zhai J, Sun J, Tao L, Zhang Y, Song Y, Hu T. Astragaloside IV enhances cisplatin chemosensitivity in hepatocellular carcinoma by suppressing MRP2. Eur J Pharm Sci 2020; 148: 105325.

13. Cui $X$, Jiang $X$, Wei $C$, Xing $Y$, Tong G. Astragaloside IV suppresses development of hepatocellular carcinoma by regulating miR-150-5p/beta-catenin axis. Environ Toxicol Pharmacol 2020; 78: 103397.

14. Negoro R, Kawai K, Ichikawa M, Deguchi S, Takayama K, Mizuguchi H. Establishment of MDR1-knockout human induced pluripotent stem cell line. Drug Metab Pharmacokinet 2020; 35: 288-296.

15. Pegram MD, Miles D, Tsui CK, Zong Y. HER2Overexpressing/Amplified Breast Cancer as a Testing Ground for Antibody-Drug Conjugate Drug Development in Solid Tumors. Clin Cancer Res 2020; 26: 775-786.

16. Zhao MM, Ge LY, Yang LF, Zheng HX, Chen G, Wu LZ, Shi SM, Wang N, Hang YP. LncRNA NEAT1/miR204/NUAK1 Axis is a Potential Therapeutic Target for Non-Small Cell Lung Cancer. Cancer Manag Res 2020 ;12:13357-13368. 